\title{
The effect of alkyl chain length on material properties of fatty-acid-functionalized amidoamine-epoxy systems
}

\author{
Arun Srikanth ${ }^{1}$, John Vergara ${ }^{1}$, Giuseppe Palmese, Cameron F. Abrams* \\ Department of Chemical and Biological Engineering, Drexel University, Philadelphia, PA 19104, USA
}

\section{A R T I C L E I N F O}

\section{Article history:}

Received 16 November 2016

Received in revised form 22 January 2017

Accepted 28 January 2017

Available online 2 February 2017

\begin{abstract}
A B S T R A C T
Polyamine crosslinkers functionalized with pendant fatty acids are commercially used in epoxy coatings in part to enhance water barrier properties for corrosion prevention. However, a systematic understanding of the links between monomer molecular structure and material properties of such systems remains elusive, which limits our ability to design newer and more environmentally friendly coating systems. In this work, the effect of $n$-alkyl chain length in fatty-acid-functionalized polyamines on crosslinked epoxyamidoamine systems is studied using a combined experimental and simulation-based approach. Both experiments and simulations show that density decreases and volumeexpansion coefficients increase with increasing pendant chain length. Furthermore, it is shown that the trends in density and coefficient of volume thermal expansion with chain length obtained from simulations are consistent with an ideal mixing approximation. Interestingly, however, the glass-transition temperature $T_{g}$ is found to be insensitive to chain length. Molecular simulations reveal that increasing the alkyl chain length from four to ten carbons does not introduce new flexibility mechanisms to the dense thermosets, which explains the $T_{g}$ insensitivity. This work demonstrates a new way to significantly decrease the density of a thermoset polymer without compromising desirable properties such as high $T_{g}$ and low coefficient of thermal expansion, and therefore may provide sounder rationale for molecular-based design of epoxy-based coatings.
\end{abstract}

(c) 2017 Elsevier Ltd. All rights reserved.

\section{Introduction}

Epoxy networks have a wide range of applications and are used as castings, encapsulating materials, adhesives, laminates and in coating formulations [1,2]. They can be made suitable for both high and low temperature applications ranging from $-50{ }^{\circ} \mathrm{C}$ to $+120^{\circ} \mathrm{C}$ [3]. Epoxies are typically cured with crosslinkers to form thermosetting polymers which possess enhanced thermomechanical properties, adhesive strength, viscosity, glass transition temperature and corrosion resistance [3]. The properties of a thermosetting polymer critically depend on the type of crosslinker used and specific crosslinkers are chosen to suit specific applications [2].

Among various crosslinkers, amidoamines are preferred in applications requiring moisture resistance, and also cause less skin irritation compared to polyamines [4,5]. Amidoamines have been in use since the late 19th century [1] and are key ingredients in the commercially available formulations Ancamide 500-507 and Ancamide 2029 [6]. However, these

\footnotetext{
* Corresponding author.

E-mail addresses: ss3763@drexel.edu (A. Srikanth), john.henry.vergara@drexel.edu (J. Vergara), grp47@drexel.edu (G. Palmese), cfa22@drexel.edu (C.F. Abrams).

${ }^{1}$ Equal contributions.
} 
formulations are poorly structurally characterized and consequently very little is known about their structure-property relationships. This limits our ability to design improved crosslinking agents. A key molecular feature of these formulations, however, are pendant $n$-alkyl fatty-acid chains that cap some primary amine sites that could otherwise crosslink. The effects of alkyl chain length in these crosslinkers on the properties of the polymer and on corrosion performance is not yet known and has hitherto not been investigated. In cases where alkyl chains are part of the internal structure of crosslinkers or epoxy molecules, the effect of increasing chain length has been studied. For instance, increasing the alkyl chain length in aliphatic amine crosslinkers decreases the glass transition temperature $T_{g}$ and the storage modulus and increases the free volume of the epoxy thermoset [7]. It has also been shown that the glass transition temperature decreases with increase in alkyl chain length in non-epoxy based systems such as polyesters [8], polyetherimides [9], poly-n-alkyl methacrylates [10], p-alkyl styrenes, and $\alpha$-olefins [11]. It is therefore worth determining whether or not increasing pendant alkyl chain length in model amidoamine crosslinkers influences basic thermomechanical properties.

The purpose of this article is to report results of a combined experimental and molecular-simulations based approach to understanding the role of pendant alkyl chain length on thermomechanical properties of thermoset polymers comprising conventional epoxy networks and model amidoamine crosslinkers. Molecular dynamics (MD) simulations have proven to be useful in estimating properties of thermoset polymers and providing molecular-level rationales for structure-property relations [12-20]. The aim here is to systematically investigate this single molecular-level feature using newly synthesized pure amidoamine (AMAM) crosslinkers of varying alkyl chain lengths. These crosslinkers are cured with the diepoxy diglycidyl ether of bisphenol A (DGEBA) and the effect of alkyl chain length on the properties of the resulting thermosets are investigated. The main properties that are addressed here are density $\rho$, glass transition temperature $T_{g}$, and coefficient of volume thermal expansion $\alpha$. It is shown that an ideal-mixing approximation is sufficient to explain trends in $\rho$ and $\alpha$ with chain length. The $T_{g}$ was found to be insensitive to $n$ and molecular simulations provide detailed explanations for this behavior.

\section{Methods}

\subsection{Experimental}

The epoxy monomer considered here is diglycidyl ether bisphenol A (DGEBA). The crosslinkers considered are and amidoamines (AMAM), derived from diethylenetriamine (DETA) via attachment of one fatty acid chain to a single primary amine site. The AMAM series considered here vary in alkyl chain length $n$ from 0 to 10 , and we denote a particular AMAM with a subscript indicating the number of methylenes in the amide moiety. DGEBA and AMAM structures are shown in Fig. 1. DGEBA was acquired from Miller-Stephenson chemicals. DETA, fatty methyl esters (methyl hexanoate, methyl octanoate, methyl decanoate, methyl dodecanoate), and chloroform were acquired from Sigma Aldrich, while magnesium sulfate was acquired from BDH. Polyvinyl fluoride, PVF (Tedlar) was used as release film for making think epoxy/amine films and was acquired from DuPont. All chemicals were used as received.

\subsubsection{Synthesis of AMAM crosslinkers}

In order to synthesize AMAM crosslinkers of specified chain length, an appropriate fatty methyl ester was reacted with DETA. The synthetic scheme is shown in Fig. 2. Diethylenetriamine $(0.467 \mathrm{~mol}, 48.26 \mathrm{~g})$ was heated to $90{ }^{\circ} \mathrm{C}$ and mixed in a two-neck round-bottom flask for $30 \mathrm{~min}$. The ester was then added dropwise to the hot DETA at a 1:10 equivalent ratio

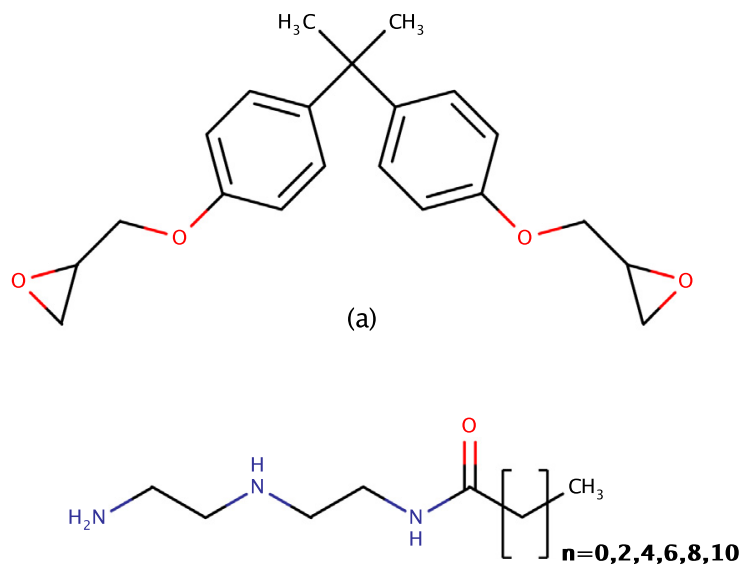

(b)

Fig. 1. Chemical structures of (a) DGEBA and (b) $\operatorname{AMAM}_{\mathrm{n}}$. 


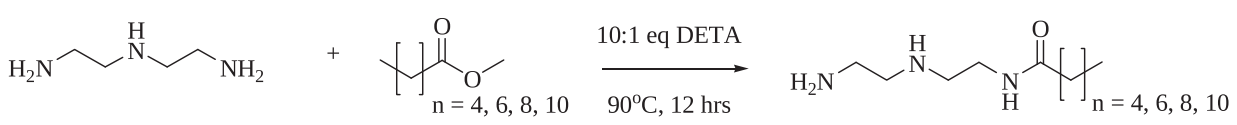

Fig. 2. Synthetic Scheme for the production of AMAM crosslinkers.

Table 1

Amine hydrogen equivalent weight (AHEW) and the molecular weight (MW) for $\mathrm{AMAM}_{n}$ systems.

\begin{tabular}{lll}
\hline Alkyl chain length $(n)$ & AHEW & MW $(\mathrm{g} / \mathrm{mol})$ \\
\hline 4 & 67.1 & 201.2 \\
6 & 76.5 & 229.4 \\
8 & 85.8 & 257.4 \\
10 & 95.2 & 285.5 \\
\hline
\end{tabular}

(10 mol of DETA: 1 mol of methyl ester) and allowed to react for $12 \mathrm{~h}$. The reaction products were diluted in $150 \mathrm{~mL}$ of chloroform and washed with $100 \mathrm{~mL}$ of water twice to remove unreacted DETA. The chloroform phase was dried with $\mathrm{MgSO}_{4}$ and roto-evaporated to isolate the desired mono-amidoamines shown in Fig. 2.

${ }^{1} \mathrm{H}$ NMR (Varian Unity Inova NMR,500 MHz) was used to confirm the $\mathrm{AMAM}_{n}$ chemical structures with a spectral window of $\pm 2000 \mathrm{~Hz}$ and 32 scans at $25^{\circ} \mathrm{C}$. ${ }^{1} \mathrm{H}$ NMR spectra are presented in the supporting information. The purity of the $\mathrm{AMAM}_{n}$ structures was found to be about $98-99 \% . \mathrm{AMAM}_{0}$ and $\mathrm{AMAM}_{2}$ could not be successfully synthesized due to cyclization of the monoamide to an imidazoline. The $\mathrm{AMAM}_{n}$ crosslinkers that were successfully synthesized $(n=4,6,8$, and 10$)$ were used in curing. The amine hydrogen equivalent weight (AHEW) and the molecular weight for the $\mathrm{AMAM}_{n}$ systems is shown in Table 1.

\subsubsection{Curing}

DGEBA was individually cured with each of the four $\operatorname{AMAM}_{n}(n=4,6,8$, and 10) crosslinkers to produce crosslinked polymer systems. The mechanism of epoxy-amine curing is shown in Fig. 3. DGEBA and crosslinker were mixed at stoichiometric ratio (one epoxy ring to one amine hydrogen) and drawn down with a 4 mil bird applicator on a PVF wrapped steel panel. The drawn film was then cured at $80^{\circ} \mathrm{C}$ for three hours and post cured at $160^{\circ} \mathrm{C}$ for one and a half hours. The resulting films were on average $100 \mu \mathrm{m}$ in thickness and were easily peeled off the PVF-wrapped panels and stored in a desiccator to prevent moisture uptake. Polymer bars were made using a homemade rectangular silicon molds where the desired polymer composition was cured at $80^{\circ} \mathrm{C}$ for three hours and post cured at $160^{\circ} \mathrm{C}$ for one and a half hours. Three samples of each system were prepared. These samples were then polished down to dimensions of $17.5 \mathrm{~mm}$ in length, $10-12 \mathrm{~mm}$ in width, and approximately $2 \mathrm{~mm}$ in thickness. These bars were then core drilled with a drill bit of $4.4 \mathrm{~mm}$ OD and the resulting cylinders were used for Thermal Mechanical Analysis (TMA). The FTIR spectra of samples shown in Fig. 4 indicate that 100\% conversion of epoxies, primary amines and secondary amines was achieved. The samples were then used for measurement of polymeric properties.

\subsubsection{Measurement of $T_{g}$, CVTE's and density}

Properties were measured for the five cured polymer compositions over three samples each. Differential scanning calorimetry (DSC; TA Q2000) was used for measuring $T_{g}$. Heat/cool scans were recorded between $40{ }^{\circ} \mathrm{C}$ to $100^{\circ} \mathrm{C}$. $T_{g}$ was measured for the polymer systems and was taken to be the inflection of the thermograms during the second heating. The second heat scan was chosen for determining $T_{g}$ because the first heat scan exhibited noise due to the relaxation of residual stresses in the polymer systems due to open curing. Thermomechanical analysis (TMA) with a temperature ramp rate of $2{ }^{\circ} \mathrm{C} / \mathrm{min}$ was used for calculating the coefficient of linear thermal expansion $\beta$, and subsequently $\alpha$, defined as

$$
\beta=\frac{1}{l} \frac{\partial l}{\partial T}=\frac{1}{3} \alpha
$$

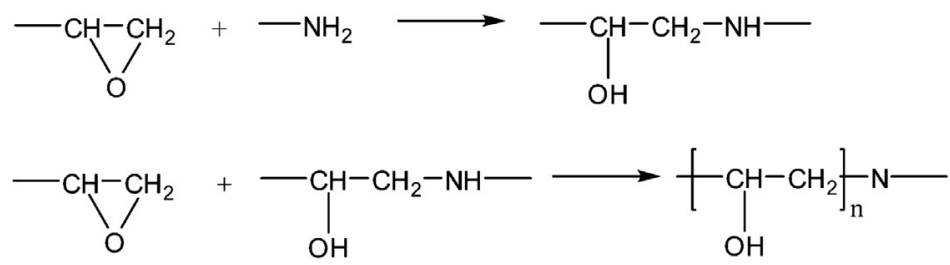

Fig. 3. Schematic of curing mechanism. 


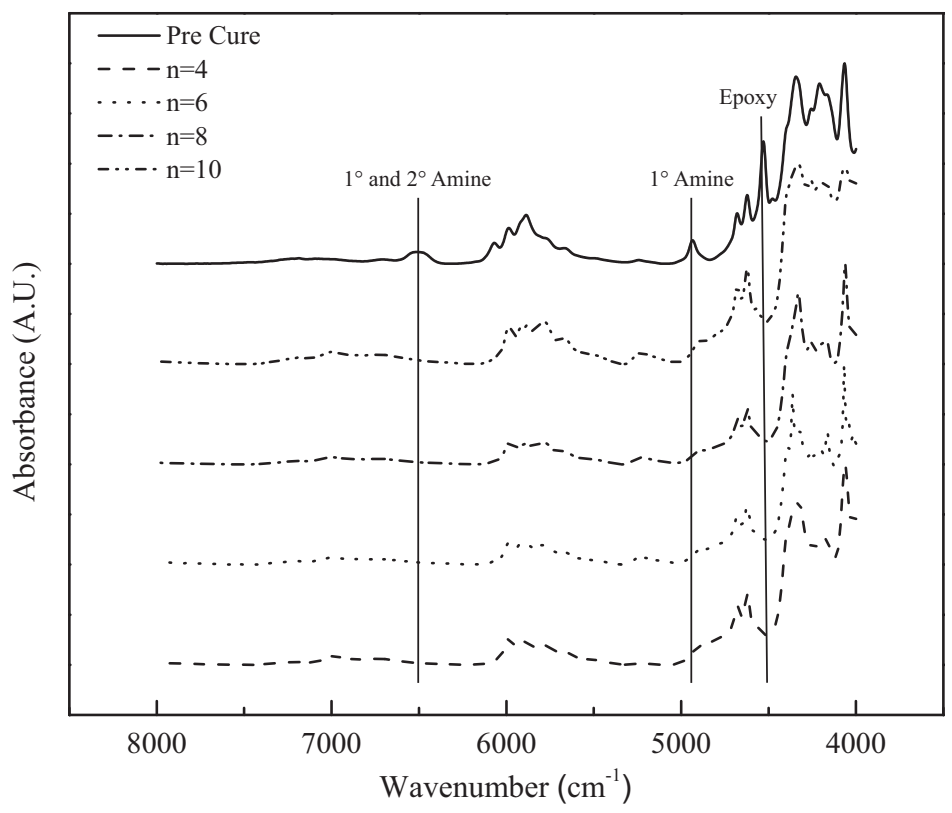

Fig. 4. N-IR Spectra of cured samples.

where the second equality holds for isotropic materials. Dimension change with temperature was measured using TA 2940 over a temperature range of $20-180^{\circ} \mathrm{C}$. The slope $\frac{\partial l}{\partial T}$ in the rubbery and glassy region was then calculated. $\beta$ for both glassy and rubbery regimes was then calculated using the length of the sample at $20^{\circ}$ and $180{ }^{\circ} \mathrm{C}$, respectively.

Density was measured using a density gradient column as described in ASTM D1505. All samples were thin films with an average thickness of $100 \mu \mathrm{m}$; these films were immersed in DI water at $23^{\circ} \mathrm{C}$ for about $10 \mathrm{~min}$ prior to exposure to the density gradient to ensure no air bubbles would form.

\subsection{Simulation}

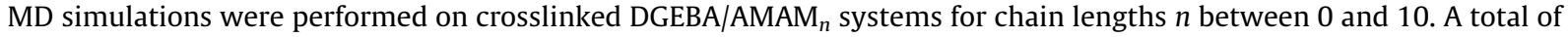
six polymer systems each consisting of 192 DGEBA molecules and 128 crosslinkers molecules of were simulated. Five independent replicas of each system composition were generated and simulated.

\subsubsection{Crosslinking protocol}

The first step in simulating a polymer system was to generate a molecular model for the epoxy and a crosslinker molecule (Fig. 1). A hydrogenated DGEBA with pre-opened epoxy rings was used in this work. The molecular models were built using avogadro [21]. After this, atom types were assigned to atoms corresponding to Generalized Amber Force Field (GAFF) [22,23]. Partial charges were assigned to the atoms using AM1-BCC method [24,25]. The force-field parameters for the topology were obtained from GAFF based on the assigned atom types. This was carried out using the antechamber suite [26]. After this step, a system or a replica of a system was created by randomly placing epoxy monomers and crosslinker molecules in a simulation cell using packmol [27].

Each system replica was then equilibrated using constant-energy MD for 50 ps followed by pressure- and temperaturecontrolled MD for $1.5 \mathrm{~ns}$. During the equilibration period, the temperature and pressure were maintained at $300 \mathrm{~K}$ and $1 \mathrm{~atm}$, respectively. After equilibration, the system was crosslinked according to the reaction mechanism shown in Fig. 3 by creating bonds between bonding partners. The bonding partners (reactive atoms) were the end carbon atoms in the epoxy and nitrogen atoms in the primary and the secondary amines in the crosslinkers. Now, with the positions of the reactive atoms as the center, the search radius $R$ was iteratively increased in steps of $2 \AA(\Delta r)$. At each iteration, potential bonding partners were identified and new bonds were created after which the hydrogen atoms were deleted. An equal reactivity assumption was employed during bond search; i.e., no distinction was made between a primary amine and a secondary amine. After each round of bond formation, atom types of the hydrogens bonded to the reactive carbon atoms were changed, the partial charges (within three bonds of the crosslinking atoms) were updated and force-field parameters were re-assigned to the newly formed topology. The system was then relaxed by employing a two stage-potential energy minimization: first, by using a Hessian-free truncated Newton algorithm followed by conjugate gradient algorithm [28] which ensured that the bond lengths of the created bonds were close to the equilibrium length. Iterations continued until the desired conversion limit was met (100\%). The crosslinking procedure described above is shown in the form a flow chart in Fig. 5. 


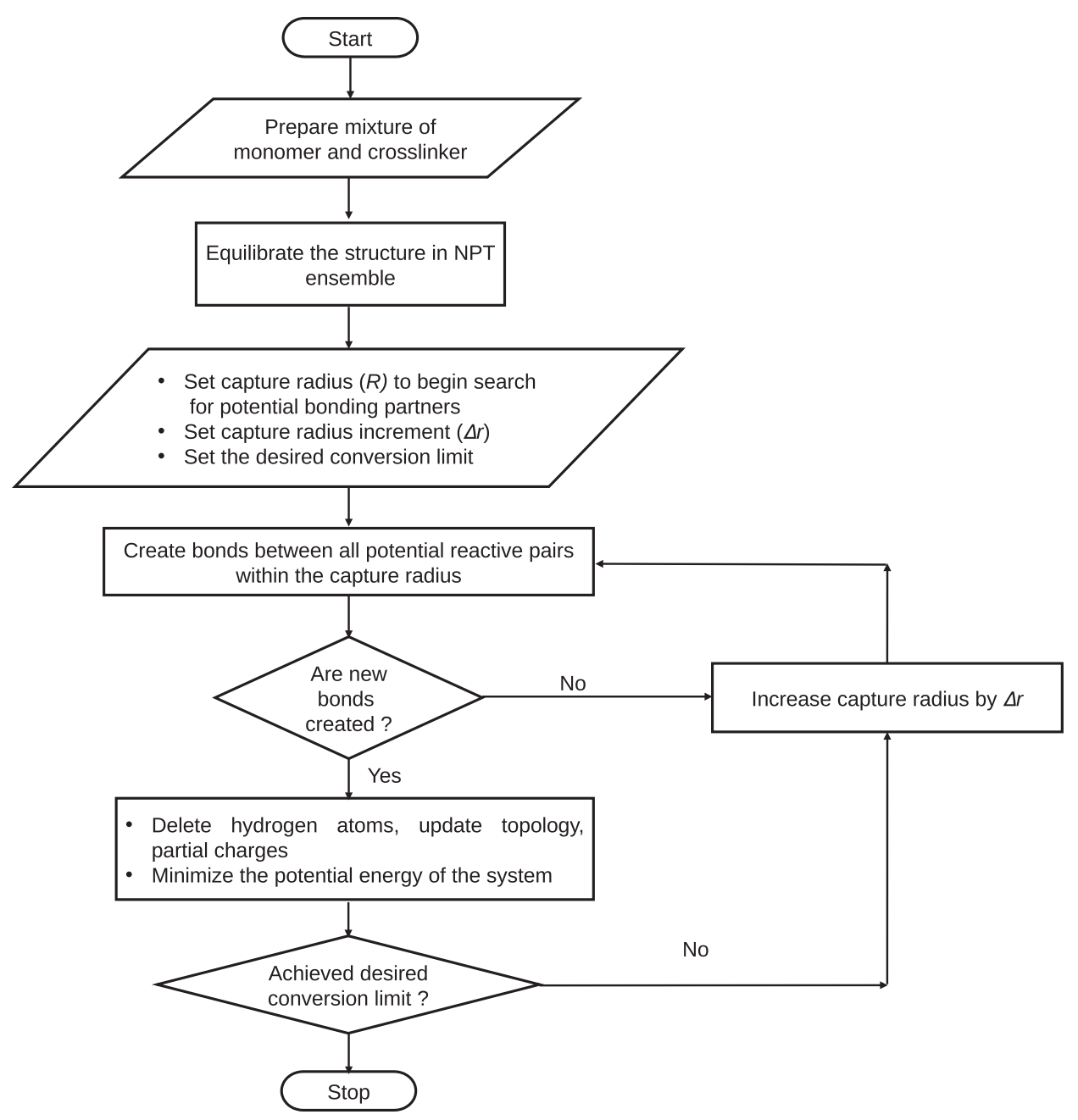

Fig. 5. Crosslinking algorithm.

The minimizations were not always successful in relaxing the bonds to the equilibrium length. In the case of an unsuccessful minimization, the replica was discarded and a new replica was prepared and subjected to equilibration and crosslinking. This process of creating a new replica of the system and subjecting it to equilibration and crosslinking was repeated until five replicas of each system were obtained for which minimizations were successful. A $100 \%$ conversion was achieved for the all crosslinked systems. For $100 \%$ conversion, our algorithm yields about $40 \%$ successfully crosslinked systems. Unsuccessful minimizations arose from particular bonding arrangements that resulted in topological restraints that prevented new bonds from shrinking to their proper lengths. These were treated as random events and were discarded. A possible way to enhance the success rate of the algorithm is to restrict the number of bonds created at each iteration, which in essence constitutes uniformly distributing the bond energy such that change in bond energy is almost kept constant at each iteration. However, because generation of crosslinked trials using this algorithm is very fast, the relatively high failure rate observed here did not limit the overall simulation throughput.

\subsubsection{Estimation of $T_{g}$}

To observe the glass-transition phenomenon, the crosslinked systems were subjected to a temperature ramp, i.e., they were held at a high temperature for about 6 ns and then slowly cooled down in steps of $15 \mathrm{~K}$ by carrying out an NPT simulation for $2 \mathrm{~ns}$ at each temperature which corresponds to a cooling rate of $7.5 \times 10^{9} \mathrm{~K} / \mathrm{s}$. The pressure was maintained at $1 \mathrm{~atm}$ throughout the temperature ramp. The six DGEBA/AMAM ${ }_{n}$ systems were subjected to a temperature ramp starting from $500 \mathrm{~K}$ and decreasing to $200 \mathrm{~K}$. The specific volume of the five replicas of a system was calculated every 5 ps, at each temperature. The second ns at each temperature was used to compute ensemble averages.

This average specific volume as a function of temperature for the polymer systems is shown in Fig. 6. Once the average specific volume as a function of temperature was obtained, two linear fits to this data, one fit to the glassy region and another 


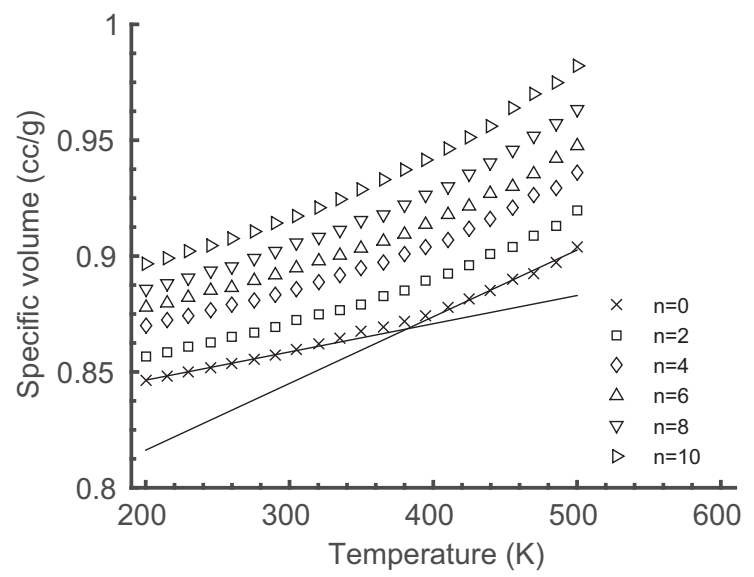

Fig. 6. Specific volume as a function of temperature. Linear fits to the rubbery and glassy region are shown for a representative system (DGEBA/AMAM 0 ).

fit to the rubbery region, were constructed. For all systems, specific volume data in the temperature range of $425-500 \mathrm{~K}$ was used for the linear fit in the rubbery region and the specific volume data in the temperature range of 200-275 $\mathrm{K}$ was used for the linear fit in the glassy region. An ordinary least squares procedure was used to construct the linear fits and $T_{g}$ is then interpreted as the intersection of the two linear fits. MATLAB [29] was used to construct the linear fits. This method of estimating $T_{g}$ is illustrated in Fig. 6. Uncertainties in $T_{g}$ were also estimated [30].

\subsubsection{Estimation of CVTE}

The CVTE $(\alpha)$ is generally defined as,

$$
\alpha=\frac{1}{v(T)}\left(\frac{\partial v}{\partial T}\right)_{p}
$$

The CVTE in the rubbery $\left(\alpha_{r}\right)$ and glassy $\left(\alpha_{g}\right)$ states of each of the DGEBA/AMAM systems were calculated using the above expression. For calculating $\alpha_{r}$, the slope of linear fit to the rubbery region was used for $\left(\frac{\partial v}{\partial T}\right)_{p}$ and specific volume at the highest temperature $(500 \mathrm{~K})$ to which the systems were subjected, was used for $v(T)$ in Eq. (2). Similarly, for calculating $\alpha_{g}$, the slope of the linear fit to the glassy region was used for $\left(\frac{\partial v}{\partial T}\right)_{p}$ and specific volume at the lowest temperature (200 K) to which the systems were subjected, was used for $v(T)$ in Eq. (2). The uncertainties in $\alpha_{g}$ and $\alpha_{r}$ were calculated using standard rules of error propagation [31].

\subsubsection{Simulation methods}

LAMMPS (Large-scale Atomic/Molecular Massively Parallel Simulator [32]) was used as the MD engine for equilibration, crosslinking, and for the temperature ramps with the following specifications. A cutoff distance of $9 \AA$ for the non-bonded interactions (electrostatics and Lennard-Jones) was used and tail corrections were used [33]. Periodic boundary conditions were used. Long range electrostatic interactions were computed using the particle-particle particle-mesh algorithm. The PPPM algorithm uses damped particle-particle interactions in real space (less than $9 \AA$ ) and particle-mesh interactions at long and short range in reciprocal space [34]. The temperature was maintained using a Nosé hoover thermostat [35,36] and pressure was maintained using a Parrinello-Rahman barostat [37]. The velocity-Verlet scheme was used for integrating the equations of motion and a timestep of $1 \mathrm{fs}$ was used. Some tools in Visual Molecular Dynamics (VMD [38]) were used for analysis of LAMMPS trajectories and visualization.

\section{Results and discussion}

In the present section the focus is on analyzing the effect of chain length in the crosslinkers on $\rho, T_{g}$, and $\alpha_{r, g}$ of crosslinked DGEBA/AMAM $_{n}$ systems obtained from the experiments and simulations.

\subsection{Effect of chain length on density}

The mass density of DGEBA/AMAM $n$ systems obtained from simulations and experiments as a function of alkyl chain length $n$ at room temperature $(300 \mathrm{~K}$ ) is shown in Fig. 7. Although quantitatively higher by a few percent (as has been observed in other simulation-experimental studies on epoxy based polymer systems $[12,17,18,14]$ ), mass densities predicted by simulations agree qualitatively with those from experiments in that density decreases with the addition of methylene 


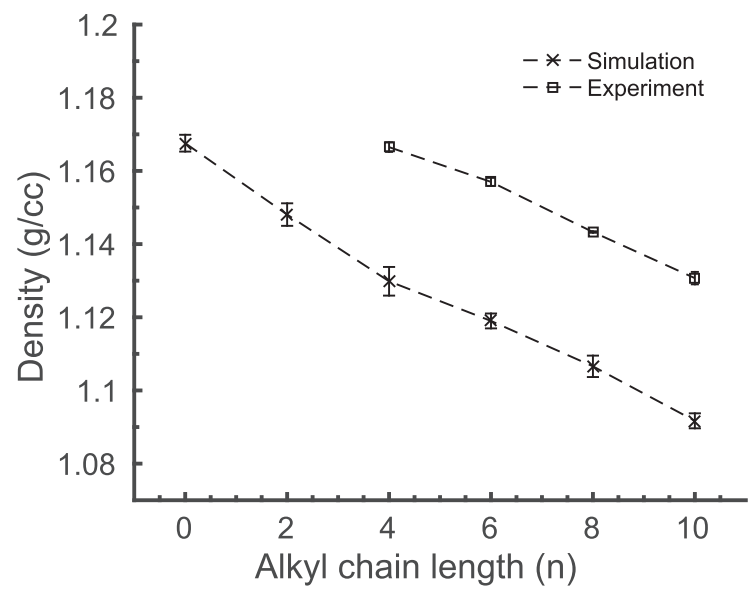

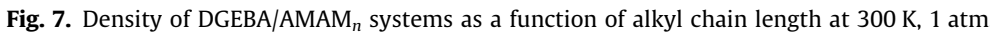

units in the AMAM crosslinker. This decrease follows a linear relation with respect to the effective volume fraction of methylene units $\Phi_{m}$, consistent with an ideal mixing approximation. That is, if one considers methylene units as solute and the rest of the polymer as solvent, then the mass density $\rho$ of the DGEBA/AMAM ${ }_{n}$ system, according to ideal mixing, is given by,

$$
\rho\left(\Phi_{m}\right)=\rho_{0}+\Phi_{m}\left(\rho_{m}-\rho_{0}\right)
$$

where $\rho_{0}$ and $\rho_{m}$ represent the density of methylene-free polymer "solvent" and the density of methylene "solute" respectively. For a given DGEBA/AMAM $n$ system, the volume fraction $\Phi_{m}=\frac{V_{n}-V_{0}}{V_{n}}$, where $V_{0}$ is the system volume of DGEBA/AMAM and $V_{n}$ is the system volume of DGEBA/AMAM $n$

A fit of Eq. (3) to the densities from simulation is shown in Fig. 8. From this fit, the pure-component density of methylene units was estimated to be $0.85 \mathrm{~g} / \mathrm{cc}$. Unsurprisingly, the density of methylene units is substantially lower than that of methylene-free polymer (DGEBA/AMAM ${ }_{0}$ ), and Eq. (3) therefore dictates that the density must decrease as $\Phi_{m}$ increases. It is to be noted that $0.85 \mathrm{~g} / \mathrm{cc}$ is in the density range of low density polymers such as PEE (polyethylethylene) and PVE (polyvinylethylene) [39] whose chemical structures are primarily composed of repeating methylenes.

\subsection{Effect of chain length on $T_{g}$}

The $T_{g}$ of crosslinked DGEBA/AMAM ${ }_{n}$ systems obtained from MD simulation and experiments is shown in Fig. 9. Both simulations and experiments show that increase in alkyl chain length have no influence on $T_{g}$ in the crosslinked DGEBA/AMAM ${ }_{n}$ systems. The $T_{g}$ obtained from simulations is higher compared to experiments due to high cooling rate employed in simulations. This can be corrected by subtracting a shift factor from the $T_{g}$ obtained from simulations which is about $33-55 \mathrm{~K}$ [40]. The $T_{g}$ obtained from simulations is then in good agreement with experiments after applying this correction.

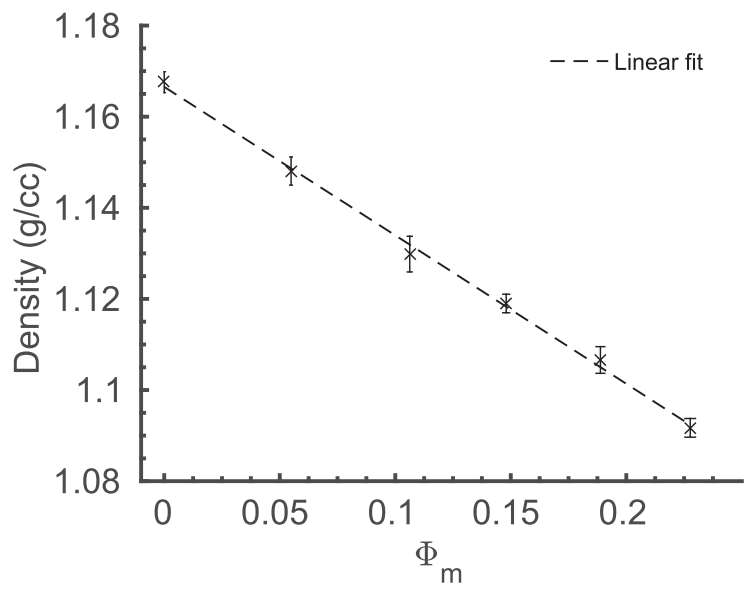

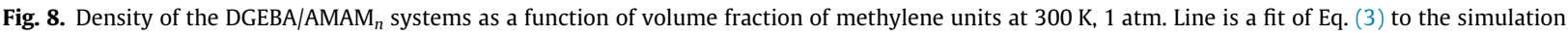
data. 


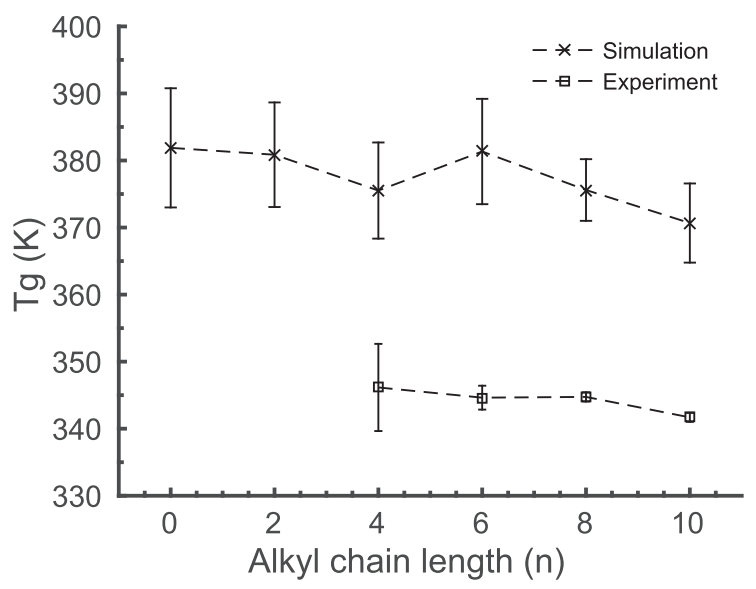

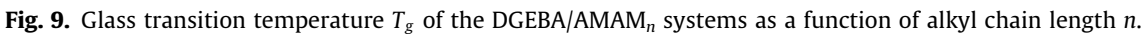

Segmental relaxation is characterized using the autocorrelation function of the end-to-end vector of the AMAM $_{n}$ crosslinker molecules, defined by

$$
c(t)=\frac{\left\langle\overrightarrow{R_{e e}}\left(t_{0}\right) \cdot \overrightarrow{R_{e e}}\left(t_{0}+t\right)\right\rangle}{\left\langle R_{e e}^{2}\right\rangle}
$$

where $\overrightarrow{R_{e e}}$ is the distance vector joining the two ends of any AMAM ${ }_{n}$. The function $c(t)$ was calculated for each of the DGEBA/ $\mathrm{AMAM}_{n}$ systems at $500 \mathrm{~K}\left(\approx T_{g}+100 \mathrm{~K}\right)$, and results appear in Fig. 10. It can be observed that there is no significant difference in the segmental relaxation regardless of chain length. This is likely because the additional rotatable $\mathrm{C}-\mathrm{C}$ bonds introduced by lengthening an $\mathrm{AMAM}_{n}$ chain are not topologically inside the crosslinked network, and therefore lengthening them does nothing to reduced steric constraints on the crosslinks. It should be further remarked that if $T_{g}$ were to decrease with increasing $n$ it would result in increased segmental relaxation as observed in other epoxy [7] and non-epoxy based thermosets [10].

This lack of increased segmental flexibility in the face of increasing chain lengths could arise because the alkyl chains are isolated from each other and essentially pinned inside the dense thermoset. From the MD simulations of the crosslinked systems, intermonomer methylene-methylene radial distribution functions (RDF's) were computed; these are shown in Fig. 11. These data clearly show that there is no correlation in the arrangement of intermonomer methylenes, and that they are not forming microphase separated domains. This is consistent with the ideal mixing approximation already demonstrated and provides some additional molecular-level rationale for the $T_{g}$ insensitivity.

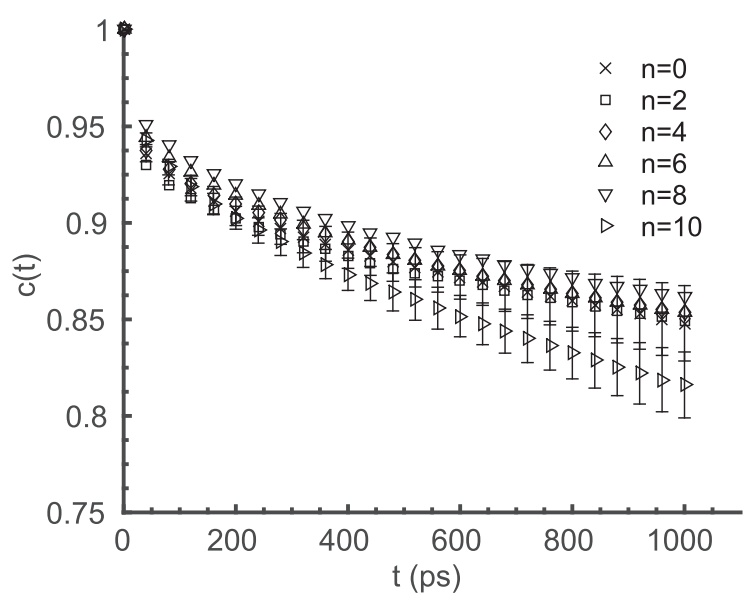

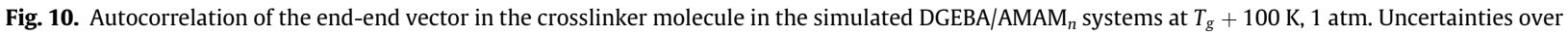
samples are shown for $n=0$ and $n=10$. 


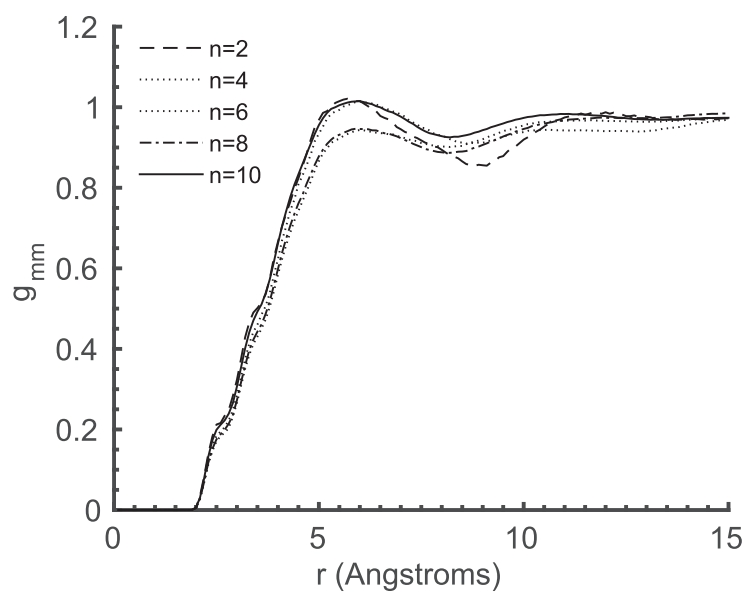

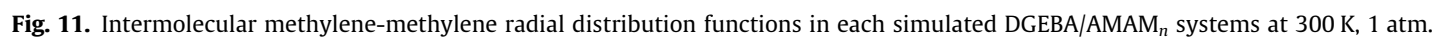

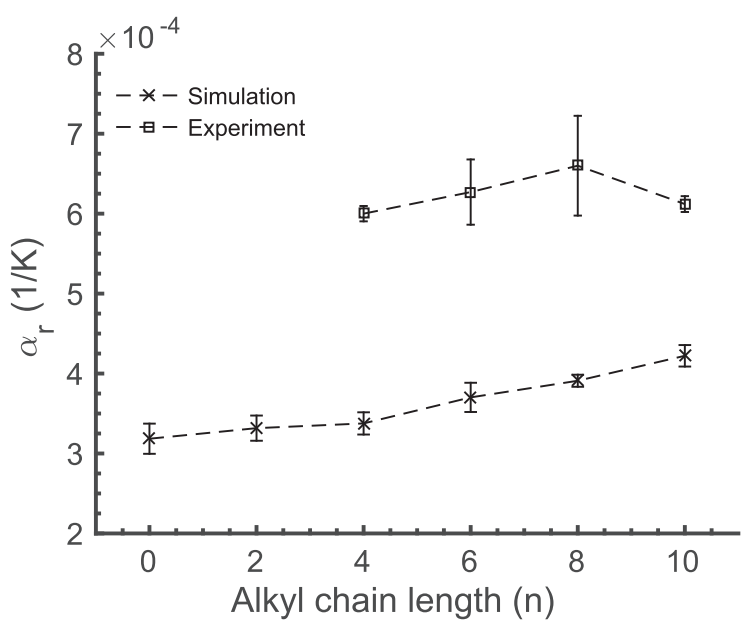

Fig. 12. Rubbery-state coefficient of volume thermal expansion $\alpha_{r}$ vs. alkyl chain length $n$ in DGEBA/AMAM ${ }_{n}$ systems at 1 atm.

\subsection{Effect of chain length on CVTE}

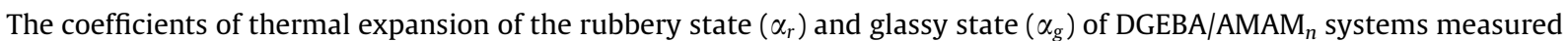
experimentally and computed from simulations are shown in Figs. 12 and 13, respectively,and all data are reported in Table 2. Both experiments and simulations agree that $\alpha_{r}>\alpha_{g}$ at each alkyl chain length $n$. Experiments do not show a clear trend and the simulations show only a weakly increasing $\alpha_{r}$ with $n$, with only a $25 \%$ increase from DGEBA/AMAM compared $_{4}$ to DGEBA/AMAM ${ }_{10}$ (Fig. 12). Experiments and simulations both show weak increases in $\alpha_{g}$ with $n$ (Fig. 13). As the number of $-\mathrm{CH}_{2}$ - groups increases with alkyl chain length, it may result in increased sub- $T_{g}$ motions that are associated with secondary relaxation ( $\beta$-relaxation) process [41]. This might provide a possible reason for the increase in $\alpha_{g}$ with $n$. Simulations systematically underpredict both $\alpha_{r}$ and $\alpha_{g}$. This may be due to the high cooling rate used in simulations or due to network imperfections in experimental polymer samples [42,43] which are otherwise absent in simulated polymer systems. It is worth mentioning that CVTEs obtained from MD simulations are mostly lower $[12,17,18,14]$ but sometimes in close agreement $[44,45]$ or even higher [46] compared to experiments.

The relationship between CVTEs $\left(\alpha_{r}, \alpha_{g}\right)$ and $n$ obtained from simulations is consistent with the ideal mixing rule defined by

$$
\alpha_{r, g}=\alpha_{0_{r, g}}+\Phi_{m}\left(\alpha_{m_{r, g}}-\alpha_{0_{r, g}}\right)
$$

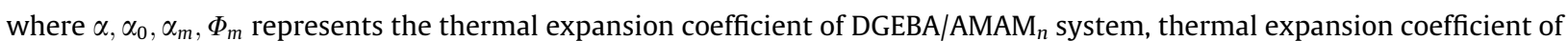
the methylene-free polymer, the thermal expansion coefficient of the methylene and the volume fraction of the methylenes 


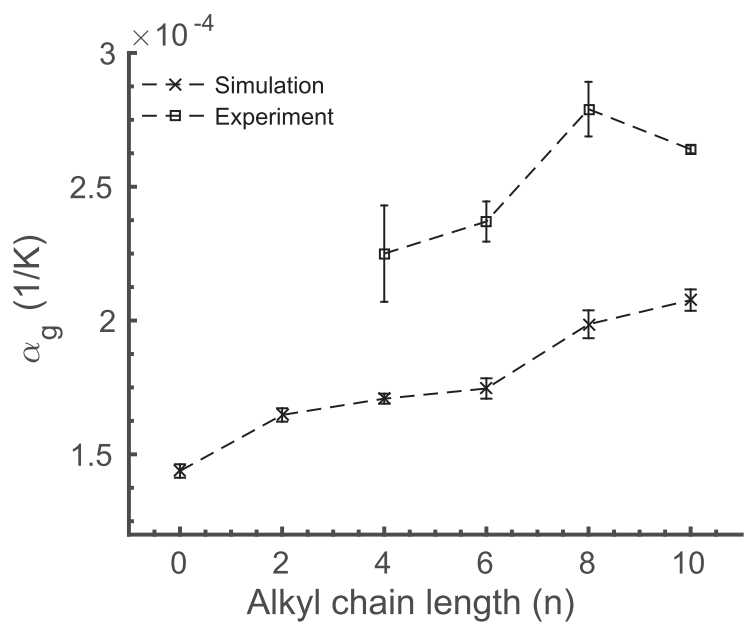

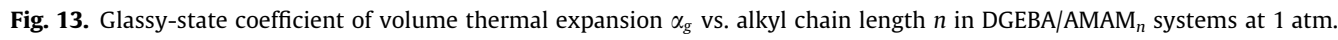

Table 2

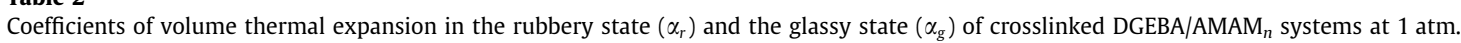

\begin{tabular}{lllll}
\hline & \multicolumn{2}{c}{$\alpha_{r} \times 10^{-4}(1 / \mathrm{K})$} & \multicolumn{2}{c}{$\alpha_{g} \times 10^{-4}(1 / \mathrm{K})$} \\
\cline { 2 - 3 } $\mathrm{n}$ & Simulation & Experiment & Simulation & - \\
\hline 0 & $3.184 \pm 0.188$ & - & $1.437 \pm 0.025$ & - \\
2 & $3.317 \pm 0.157$ & - & $1.647 \pm 0.025$ & $2.25 \pm 0.18$ \\
4 & $3.376 \pm 0.138$ & $6.00 \pm 0.096$ & $1.708 \pm 0.018$ & $2.37 \pm 0.075$ \\
6 & $3.702 \pm 0.182$ & $6.27 \pm 0.408$ & $1.746 \pm 0.038$ & $2.79 \pm 0.10$ \\
8 & $3.910 \pm 0.075$ & $6.60 \pm 0.624$ & $2.986 \pm 0.052$ & $2.64 \pm 0.015$ \\
\hline
\end{tabular}

in the polymer system respectively. The fit of Eq. (5) to the simulation data is shown in Fig. 14. The value of $\alpha_{m_{r}}$ and $\alpha_{m_{g}}$ were estimated to be $7.4 \times 10^{-4} 1 / \mathrm{K}$ and $4.15 \times 10^{-4} 1 / \mathrm{K}$ respectively. The thermal expansion coefficients of methylenes is significantly higher compared to the thermal expansion coefficients of DGEBA/AMAM ${ }_{0}$. This is expected as low molecular weight additives tend to have high thermal expansion coefficients [11] and therefore Eq. (5) stipulates that $\alpha$ must increase as $\Phi_{m}$ increases, however, the increase is not significant as observed here. Both $\alpha_{r}$ and $\alpha_{g}$ are in good agreement with the idealmixing approximation. This agreement is a consequence of methylene units being well dispersed and the absence of correlation among the methylene units in the polymer system (Fig. 11).

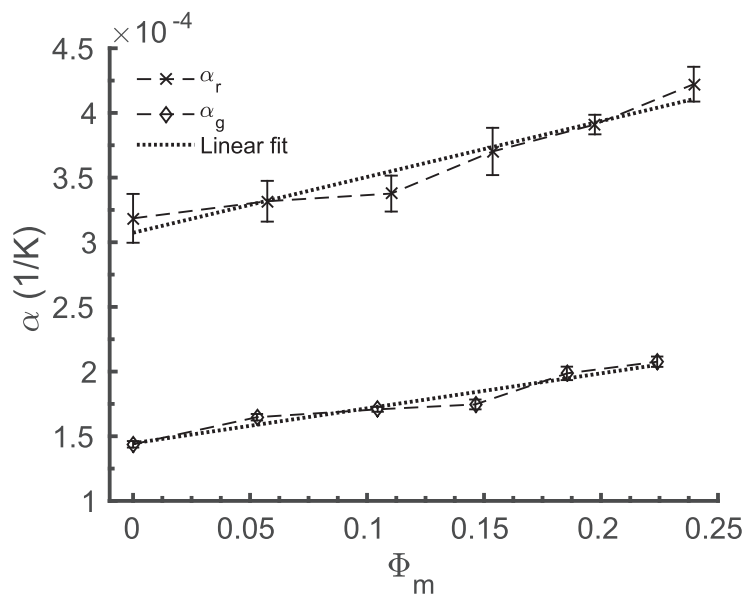

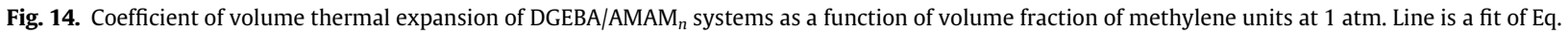
(5) to the simulation data. 


\section{Conclusions}

Amidoamine $\left(\mathrm{AMAM}_{n}\right.$ ) crosslinkers with pendant alkyl chains of four different lengths ( $n=4,6,8$, and 10 ) were synthesized and cured with DGEBA to generate novel crosslinked thermosets. In addition, MD simulations were used to study model DGEBA/AMAM ${ }_{n}$ systems for $n=0,2,4,6,8$, and 10 . Satisfactory agreement between simulations and experiments was observed for densities, glass transition temperatures, and coefficients of volume thermal expansion. The trend in density and CVTE with $n$ followed an ideal-mixing approximation based on partitioning into solvent polymer and solute alkyl chains fairly robustly, which was further corroborated by the lack of methylene-methylene correlations seen in radial distribution functions. However, it was found that $T_{g}$ was largely insensitive to $n$. This indicates that although the pendant alkyl chains affect density and CVTE, they do not plasticize these thermosets. This observation was further corroborated by end-to-end autocorrelation functions of the $\mathrm{AMAM}_{n}$ monomers from MD which indicated no increase in segmental flexibilty with $n$. These findings are relevant for the design of novel crosslinking molecules in thermosets where alkylation is anticipated to both lower density and potentially improve moisture barrier properties without compromising $T_{g}$ or $\alpha$, and suggests that pendant alkylation is a desirable approach.

\section{Acknowledgement}

Funding from the US Army Research Laboratory (W911NF-13-2-0046) is gratefully acknowledged. Simulations were performed in part on equipment in the Drexel University Research Computing Facility and in part thanks to the US DoD highperformance computing modernization program (HPCMP).

\section{References}

[1] X.M. Chen, B. Ellis, Coatings and Other Applications of Epoxy Resins, Springer, Netherlands, Dordrecht, 1993, pp. 303-325.

[2] Fan-Long Jin, Xiang Li, Soo-Jin Park, Synthesis and application of epoxy resins: a review, J. Ind. Eng. Chem. 29 (2015) 1-11.

[3] S.J. Shaw, Additives and Modifiers for Epoxy Resins, Springer, Netherlands, Dordrecht, 1993, pp. 117-143.

[4] A. Marcilla, M. Beltrán, 5 - Mechanisms of plasticizers action, in: George Wypych (Ed.), Handbook of Plasticizers, second ed., William Andrew Publishing, Boston, 2012, pp. 119-133.

[5] S. Zheng, P.G. Patel, G.A. Vedage, E.J. Tijsma, G.S. Lal, Epoxy liquid curing agent compositions, April 2 2015. WO Patent App. PCT/US2014/058,156.

[6] E.W. Flick, Epoxy Resins, Curing Agents, Compounds, and Modifiers: An Industrial Guide Pdl, (Plastics Design Library) Handbook, Elsevier Science, 2012.

[7] John S. Nakka, K.M.B. Jansen, L.J. Ernst, Effect of chain flexibility in the network structure on the viscoelasticity of epoxy thermosets, J. Polym. Res. 18 (6) (2011) 1879-1888.

[8] Dirk Schmaljohann, Liane Huler, Petra Ptschke, Brigitte I. Voit, Ton J.A. Loontjens, Modification with alkyl chains and the influence on thermal and mechanical properties of aromatic hyperbranched polyesters, Macromol. Chem. Phys. 201 (1) (2000) 49-57.

[9] Rui Pan, Wei Zhao, Tao Zhou, Aiming Zhang, Effect of alkyl side chain length on the properties of polyetherimides from molecular simulation combined with experimental results, J. Polym. Sci. Pol. Phys. 48 (5) (2010) 595-599.

[10] K.L. Ngai, S. Etienne, Z.Z. Zhong, D.E. Schuele, Effect of alkyl chain spacer length on the dynamics of glass transition in side-chain liquid-crystalline polymers, Macromolecules 28 (19) (1995) 6423-6431.

[11] J. Mark, Physical Properties of Polymers, Cambridge University Press, 2004.

[12] Nipun J. Soni, Po-Han Lin, Rajesh Khare, Effect of cross-linker length on the thermal and volumetric properties of cross-linked epoxy networks: a molecular simulation study, Polymer 53 (4) (2012) 1015-1019.

[13] Po-Han Lin, Rajesh Khare, Local chain dynamics and dynamic heterogeneity in cross-linked epoxy in the vicinity of glass transition, Macromolecules 43 (15) (2010) 6505-6510.

[14] Po-Han Lin, Rajesh Khare, Molecular simulation of cross-linked epoxy and epoxyposs nanocomposite, Macromolecules 42 (12) (2009) $4319-4327$.

[15] Changwoon Jang, Majid Sharifi, Giuseppe R. Palmese, Cameron F. Abrams, Crosslink network rearrangement via reactive encapsulation of solvent in epoxy curing: a combined molecular simulation and experimental study, Polymer 55 (16) (2014) 3859-3868.

[16] Robert M. Elder, Jan W. Andzelm, Timothy W. Sirk, A molecular simulation study of the glass transition of cross-linked poly(dicyclopentadiene) networks, Chem. Phys. Lett. 637 (2015) 103-109.

[17] Alexey A. Gavrilov, Pavel V. Komarov, Pavel G. Khalatur, Thermal properties and topology of epoxy networks: a multiscale simulation methodology, Macromolecules 48 (1) (2015) 206-212.

[18] Baris Demir, Tiffany R. Walsh, A robust and reproducible procedure for cross-linking thermoset polymers using molecular simulation, Soft Matter 12 (2016) 2453-2464.

[19] Chunyu Li, Alejandro Strachan, Molecular dynamics predictions of thermal and mechanical properties of thermoset polymer epon862/detda, Polymer 52 (13) (2011) 2920-2928.

[20] Gregory M. Odegard, Benjamin D. Jensen, S. Gowtham, Jianyang Wu, Jianying He, Zhiliang Zhang, Predicting mechanical response of crosslinked epoxy using reaxff, Chem. Phys. Lett. 591 (2014) 175-178.

[21] Marcus Hanwell, Donald Curtis, David Lonie, Tim Vandermeersch, Eva Zurek, Geoffrey Hutchison, Avogadro: an advanced semantic chemical editor, visualization, and analysis platform, J. Cheminfo. 4 (1) (2012) 17+.

[22] Junmei Wang, Romain M. Wolf, James W. Caldwell, Peter A. Kollman, David A. Case, Development and testing of a general amber force field, J. Comput. Chem. 25 (9) (2004) 1157-1174. July.

[23] Junmei Wang, Wei Wang, Peter A. Kollman, David A. Case, Automatic atom type and bond type perception in molecular mechanical calculations, J. Mol. Graph. Model. 25 (2) (2006) 247-260. October.

[24] Araz Jakalian, David B. Jack, Christopher I. Bayly, Fast, efficient generation of high-quality atomic charges. AM1-BCC model: II. Parameterization and validation, J. Comput. Chem. 23 (16) (2002) 1623-1641. December.

[25] Araz Jakalian, Bruce L. Bush, David B. Jack, Christopher I. Bayly, Fast, efficient generation of high-quality atomic charges. am1-bcc model: I. Method, J. Comput. Chem. 21 (2) (2000) 132-146.

[26] D.A. Case, J.T. Berryman, R.M. Betz, T.E D.S. Cerutti, Cheatham III, T.A. Darden, R.E. Duke, T.J. Giese, H. Gohlke, A.W. Goetz, S. Izadi, N. Homeyer, P. Janowski, J. Kaus, A. Kovalenko, T.S. Lee, S. LeGrand, P. Li, T. Luchko, R. Luo, B. Madej, K.M. Merz, G. Monard, P. Needham, H. Nguyen, H.T. Nguyen, I. Omelyan, A. Onufriev, D.R. Roe, A. Roitberg, R. Salomon-Ferrer, C.L. Simmerling, W. Smith, J. Swails, R.C. Walker, J. Wang, R.M. Wolf, X. Wu, D.M. York, P. A. Kollman, Amber 2015, University of California, San Francisco, 2015.

[27] L. Martnez, R. Andrade, E.G. Birgin, J.M. Martnez, Packmol: A package for building initial configurations for molecular dynamics simulations, J. Comput. Chem. 30 (13) (2009) 2157-2164. 
[28] J. Nocedal, S.J. Wright, Numerical Optimization, second ed., Springer, New York, 2006.

[29] MATLAB. 8.4.0.150421 (R2014b), The MathWorks Inc., Natick, Massachusetts, United States, September 15, 2014

[30] Lowell M. Schwartz, Robert I. Gelb, Statistical uncertainties of end points at intersecting straight lines, Anal. Chem. 56 (8) (1984) 1487-1492.

[31] Gerhard Bohm, Günter Zech, Introduction to Statistics and Data Analysis for Physicists, Deutsches Elektronen-Synchrotron, Hamburg, 2010.

[32] Steve Plimpton, Fast parallel algorithms for short-range molecular dynamics, J. Comput. Phys. 117 (1) (1995) 1-19.

[33] H. Sun, Compass: an ab initio force-field optimized for condensed-phase applications overview with details on alkane and benzene compounds, J. Phys. Chem. B 102 (38) (1998) 7338-7364.

[34] Markus Deserno, Christian Holm, How to mesh up ewald sums. I. A theoretical and numerical comparison of various particle mesh routines, J. Chem. Phys. 109 (18) (1998) 7678-7693.

[35] S. Shuichi Nosé, A unified formulation of the constant temperature molecular dynamics methods, J. Chem. Phys. 81 (1) (1984) 511-519.

[36] William G. Hoover, Canonical dynamics: equilibrium phase-space distributions, Phys. Rev. A 31 (1985) 1695-1697. Mar.

[37] Shuichi Nosé, M.L. Klein, Constant pressure molecular dynamics for molecular systems, Mol. Phys. 50 (5) (1983) $1055-1076$.

[38] William Humphrey, Andrew Dalke, Klaus Schulten, VMD - Visual Molecular Dynamics, J. Mol. Graph. 14 (1996) $33-38$.

[39] L.J. Fetters, D.J. Lohse, R.H. Colby, Chain Dimensions and Entanglement Spacings, Springer, New York, NY, 2007, pp. $447-454$.

[40] M.D. Ediger, C.A. Angell, Sidney R. Nagel, Supercooled liquids and glasses, J. Phys. Chem. 100 (31) (1996) 13200-13212.

[41] R.N. Haward, The Physics of Glassy Polymers, Springer, Netherlands, 2012.

[42] Huaxing Zhou, Jiyeon Woo, Alexandra M. Cok, Muzhou Wang, Bradley D. Olsen, Jeremiah A. Johnson, Counting primary loops in polymer gels, Proc. Natl. Acad. Sci. USA 109 (47) (2012) 19119-19124.

[43] Paul J. Flory, Network topology and the theory of rubber elasticity, Br. Polym. J. 17 (2) (1985) 96-102.

[44] Hai Bo Fan, Matthew M.F. Yuen, Material properties of the cross-linked epoxy resin compound predicted by molecular dynamics simulation, Polymer 48 (7) (2007) 2174-2178.

[45] Chaofu Wu, Weijian Xu, Atomistic molecular modelling of crosslinked epoxy resin, Polymer 47 (16) (2006) 6004-6009.

[46] Pavel V. Komarov, Chiu Yu-Tsung, Chen Shih-Ming, Pavel G. Khalatur, Peter Reineker, Highly cross-linked epoxy resins: an atomistic molecular dynamics simulation combined with a mapping/reverse mapping procedure, Macromolecules 40 (22) (2007) 8104-8113. 